\title{
DNS OF FALLING DROPLETS IN A VERTICAL CHANNEL
}

\author{
NÉSTOR BALCÁZAR ${ }^{1,2}$, JESÚS CASTRO ${ }^{1}$, JORGE CHIVA $^{1}$ \& ASSENSI OLIVA $^{1}$ \\ ${ }^{1}$ Heat and Mass Transfer Technological Center (CTTC), Universitat \\ Politècnica de Catalunya - BarcelonaTech (UPC) ETSEIAT, \\ Colom Terrassa (Barcelona), Spain. \\ 2 Termo Fluids, S.L., Avda Jacquard 97 1-E, Terrassa \\ (Barcelona), Spain
}

\begin{abstract}
This paper presents Direct Numerical Simulation (DNS) of the falling motion of single and multiple deformable drops in a vertical channel. A systematic study of the wall effect on the motion of single drop is performed for Eötvös number $(0.5 \leq \mathrm{Eo} \leq 5)$, Morton number $\left(10^{-3} \leq \mathrm{M} \leq 10^{-8}\right)$, and confinement ratio $\mathrm{CR}=2$. Second, the gravity-driven motion of multiple drops and their interactions are studied in a periodic vertical channel for $\mathrm{CR}=4$. These simulations are performed using a multiple marker level-set methodology, integrated in a finite-volume framework on a collocated unstructured grid. Each droplet is described by a level-set function, which allows capturing multiple interfaces in the same control volume, avoiding the numerical merging of the droplets. Numerical algorithms for fluid motion and interface capturing have been developed in the context of the finite-volume and level-set methodology, surface tension is modeled by means of the continuous surface force approach, and the pressure-velocity coupling is solved using a fractional-step projection method. DNS of single drop shows that they migrate to the symmetry axis of the channel when the Reynolds number is low, following a monotonic approach or damped oscillations according to the dimensionless parameters. If Eötvös number increases, stronger oscillations around the symmetry axis are observed. Simulations of multiple drops show that the collision of two drops follows the drafting-kissing tumbling (DKT) phenomenon. Deformable drops do not collide with the wall, whereas DKT phenomenon in the droplet swarm leads to the formation of groups which move through the center of the channel.
\end{abstract}

Keywords: conservative level-set method, DNS, drops, interface capturing, multiphase flow, multiple marker, surface tension, vertical channel

\section{INTRODUCTION}

Falling droplets play an important role in natural and technological processes, for instance: industrial jet burners, unit operations in chemical engineering such as flotation, liquid-liquid extraction, and absorption columns used for scrubbing of a polluted gas. Thus, the ability to understand and predict this type of flows is mandatory to achieve a reliable design and scale-up of the systems mentioned above. Furthermore, besides these practical applications, there are complex aspects of scientific interest such as the dynamics and stability of falling droplets, the generated flow field structures, and the hydrodynamics interaction of multiple droplets, which motivate us to study these phenomena from a fundamental point of view.

The deformation and interaction of falling droplets is a challenging fluid mechanics problem which leads to a complex and highly non-linear mathematical model, thus, using analytical solutions is restricted only to the simplest cases, whereas experiments can be difficult to implement due to limitations in optical instruments. On the other hand, the advances in supercomputers have promoted the use of high-performance computing (HPC) and direct numerical simulation (DNS) of interfacial flows as an emerging methodology to perform non-invasive and controlled experiments of droplet dynamics, with accurate control of the fluid particle size distribution, droplet deformability, coalescence, and flow conditions [1]. In the last decades, multiple methods have been introduced to model free surface and interfacial flows, for instance: the front tracking (FT) method [2], lattice Boltzmann method [3], 
level-set (LS) methods [4-9], volume-of-fluid (VOF) methods [10], and coupled VOF/LS methods [11-13]. In these methods, the interface is captured using an Eulerian framework (VOF, LS, VOF/LS) or using a Lagrangian framework (FT), whereas two-phase flow is treated as a single flow with a smooth variation of physical properties across the fluid interface.

In this context, a great deal of experimental research on the motion and deformation of droplets has been presented by Ref. [14]. Approximate analytical solutions have been reported in [15-18] that studied the breakup of a liquid drop accelerated by a constant body force for small density ratio using the front-tracking method; Ref. [3] reported simulation of falling droplet using the lattice Boltzmann method; and Ref. [19] presented a numerical study of the lateral migration of one drop in Poiseuille flow. Recently, in Ref. [20], the wall effects on gravity-driven falling droplets in a two-wall vertical duct using the front-tracking method was researched. To the best of the authors' knowledge, there are not yet computational studies of the gravity-driven falling motion of single and multiple droplets in a vertical channel with the square section. Moreover, since previous numerical research about the motion of single and multiple droplets has been performed using the front-tracking method [2], the ability, stability, and accuracy of new methodologies such as the multiple marker CLS method introduced in Ref. [4] need to be proven. It is the purpose of this work to perform a systematic study of the dynamics of single and multiple droplets falling in a vertical channel, with the aid of the multiple marker CLS method introduced in Ref. [4]. An advantage of the CLS method [5, 8] is that accumulation of mass conservation error inherent to standard level-set formulations is avoided. However, one drawback of CLS, LS and VOF/LS methods is the numerical and potentially unphysical merging of the fluid interfaces. The multiple marker CLS methodology overcomes the last problem, while the mass conservation errors are minimized. Indeed, the present research is a further step to explore the complex interaction of multiple droplets without numerical merging of droplets, keeping constant the number of fluid particles, and taken into account the physics of droplet collisions.

The present paper is organized as follows: The mathematical formulation and numerical methods are reviewed in Section 2. Numerical experiments are presented in Section 3. Finally, conclusions and future work are remarked in Section 4.

\section{MATHEMATICAL MODEL AND NUMERICAL METHODS}

\subsection{Incompressible two-phase flow}

The mathematical model used in this work has been introduced by [4-6], and here is reviewed for the sake of completeness. The Navier-Stokes equations for the droplet fluid in $\Omega_{\mathrm{d}}$ and continuous fluid in $\Omega_{\mathrm{c}}$ are written in the conservative form, into a one set of equations in the global domain $\Omega=\Omega_{\mathrm{d}} \cup \Omega_{\mathrm{c}}$, with a singular source term for the surface tension force at the interface $\Gamma[4,5]$ :

$$
\begin{gathered}
\frac{\partial}{\partial \mathrm{t}}(\rho \boldsymbol{v})+\nabla \cdot(\rho \boldsymbol{v} \boldsymbol{v})=-\nabla p+\nabla \cdot \mu\left(\nabla \boldsymbol{v}+(\nabla \boldsymbol{v})^{T}\right)+\left(\rho-\rho_{0}\right) \boldsymbol{g}+\boldsymbol{f}_{\sigma} \delta_{\Gamma} . \\
\nabla \cdot \boldsymbol{v}=0 .
\end{gathered}
$$

where $v$ is the velocity field, $p$ denotes the pressure field, $\rho$ is the fluid density, $\mu$ is the dynamic viscosity, $\boldsymbol{g}$ is the gravity acceleration, subscripts $d$ and $c$ are used for the dispersed and continuous fluids, respectively, and $\delta_{\Gamma}$ is the Dirac delta function concentrated at the 
interface $\Gamma$. Since the domain is periodic along the $y$-axis direction, $-\rho_{0} g$ is included in the Navier-Stokes equations $[4,7]$, with $\rho_{0}=\left(V_{\Omega}\right)^{-1} \int\left(\rho_{d} \phi_{d}+\rho_{d}\left(1-\phi_{d}\right)\right) d V$ and $\phi_{d}$ a global level-set function introduced in eqn. (8) $[4,6]$, in order to prevent the acceleration of the flow field in the downward vertical direction by the action of $\boldsymbol{g}[4,7]$. Density and viscosity are constant at each fluid-phase with a jump discontinuity at the interface:

$$
\rho=\rho_{d} H_{d}+\rho_{c}\left(1-H_{d}\right) \mu=\mu_{d} H_{d}+\mu_{c}\left(1-H_{d}\right) .
$$

where $H_{d}$ is defined as the Heaviside step function that is 1 at fluid $d$ and 0 elsewhere. A continuous treatment of physical properties is adopted at the discretized level, in order to avoid numerical instabilities at the interface, according to the multiple marker CLS method introduced in Refs [4, 7].

\subsection{Interface capturing and surface tension}

Present computations are performed in the context of the multiple marker CLS method introduced in Ref [4]. In this method, the concept of multiple marker [4, 12] is combined with the conservative level-set method (CLS) [8] developed by Balcázar et al. [5] for interface capturing on unstructured grids. Moreover, each fluid particle is represented by a CLS function in order to avoid their numerical merging $[4,6]$. The interface of the ith droplet is represented by the 0.5 iso-surface of a regularized indicator function $\phi_{i}[5,8]$, where the sub-index $\mathrm{i}=1,2, \ldots, \mathrm{n}_{d}$, and $\mathrm{n}_{d}$ is the total number of droplets in the dispersed phase. Since the velocity field is incompressible, it is possible to write the ith interface transport equation in conservative form, as follows:

$$
\frac{\partial \phi_{i}}{\partial t}+\nabla \cdot \phi_{i} v=0
$$

With the aim to keep a sharp and constant interface profile, a re-initialization equation is solved [8]:

$$
\frac{\partial \phi_{i}}{\partial \tau}+\nabla \cdot \phi_{i}\left(1-\phi_{i}\right) \boldsymbol{n}_{i}=\nabla \cdot \varepsilon \nabla \phi_{i}
$$

This equation is advanced in pseudo-time $\tau$, up to achieve the steady state. The compressive term, $\phi_{i}\left(1-\phi_{i}\right) \boldsymbol{n}_{\boldsymbol{i}}$, with $\boldsymbol{n}_{\boldsymbol{i}}$ evaluated at $\tau=0$, forces the CLS function $\phi_{i}$ to be compressed onto the interface along the normal vector $\boldsymbol{n}_{i}$. The diffusion term $\nabla \bullet \varepsilon \nabla \phi_{i}$ ensures the CLS profile remains of characteristic thickness $\varepsilon=0.5 h^{0.9}$ with h defined as the grid size [5, 7].

Geometrical properties of the interface such as normal vector $\boldsymbol{n}_{i}$, and curvature $\kappa_{i}$, are obtained from the CLS function, as follows:

$$
\boldsymbol{n}_{i}=\frac{\nabla \phi_{i}}{\left\|\nabla \phi_{i}\right\|}, \kappa_{i}=-\nabla \cdot \boldsymbol{n}_{i} .
$$

Surface tension force $f_{\sigma} \delta_{\Gamma}$ is calculated using the continuous surface force model (CSF) introduced by Brackbill et al. [21]. This model has been extended to the multiple marker CLS approach in Refs [4, 6], as follows:

$$
\boldsymbol{f}_{\sigma} \delta_{\Gamma}=\sum_{i=1}^{n_{d}}\left(\boldsymbol{f}_{\sigma} \delta_{\Gamma}\right)_{i}=\sum_{i=1}^{n_{d}} \sigma \kappa_{i}\left(\phi_{i}\right) \nabla \phi_{i} .
$$


Following the work of Balcázar et al. [4, 6], fluid properties in eqn. (3) are regularized using a global CLS function $H_{d}=\phi_{d}$, to avoid numerical instabilities at the interface:

$$
\phi_{d}=\max \left\{\phi_{1}, \phi_{2}, \ldots, \phi_{n_{d}}\right\} \text {. }
$$

\subsection{Numerical methods}

The numerical discretization has been introduced in our previous works [4-6], and here it is reviewed for the sake of completeness. The Navier-Stokes equations, eqns (1 and 2), and interface capturing equations, eqns ( 4 and 5), are solved using a finite-volume discretization of $\Omega$ on a collocated unstructured grid [5,6], where both vector and scalar variables $(p, v, \mu, \rho)$ are stored in the cell centroids. Following Refs $[5,6]$, the convective term of both momentum equation (eqn. (1)) and interface transport equation (eqn. (4)) is explicitly calculated approximating the fluxes at cell faces with a Total Variation Diminishing (TVD) Superbee flux limiter scheme $[4,5]$, in order to suppress numerical oscillations at the discontinuities and to minimize the numerical diffusion. TVD schemes (Superbee, Van-Leer) designed for unstructured grids in Refs [5, 6] have proved to be robust and accurate to solve two-phase flows with high-density ratio $(O(1000))$ and high-viscosity ratio $(O(6000))$, as well as flows with high Reynolds numbers involved in the motion of wobbling bubbles with topology changes [7, 11]. Diffusive terms are centrally differenced unless otherwise stated, gradients are computed at cell centroids using the least-squares method [5], whereas a distance-weighted linear interpolation is used to find the cell face values of physical properties, gradients and interface normals [5]. A central difference scheme is used to discretize both compressive and diffusive terms of the re-initialization equation, eqn. (5) [5]. A standard fractional step projection method originally introduced by [23], is used for the resolution of the pressure-velocity coupling:

$$
\frac{\rho \boldsymbol{v}^{*}-\rho^{n} \boldsymbol{v}^{n}}{\Delta t}=A^{n}+\boldsymbol{D}^{n}+\left(\rho-\rho_{0}\right) \boldsymbol{g}+\sum_{i=1}^{n_{d}} \sigma \kappa_{i}\left(\phi_{i}\right) \nabla \phi_{i}, \frac{\rho \boldsymbol{v}^{n+1}-\rho \boldsymbol{v}^{*}}{\Delta t}=-\nabla p
$$

where $\boldsymbol{A}=-\nabla_{h} \cdot(\rho \boldsymbol{v} \boldsymbol{v})$ and $\boldsymbol{D}=\nabla_{h} \cdot \mu\left(\nabla \boldsymbol{v}+(\nabla \boldsymbol{v})^{T}\right)$ are explicitly evaluated, $(\nabla \boldsymbol{v})^{T}$ is calculated by the least-squares method [5]. The combination of the incompressibility constraint $\nabla_{h} \cdot \boldsymbol{v}^{n+1}=0$ with the corrector step in eqn. (9), leads to the Poisson equation for the pressure field, which is solved using a preconditioned conjugate gradient method:

$$
\nabla_{h} \cdot\left(\frac{1}{\rho} \nabla_{h} p\right)=\frac{1}{\Delta t} \nabla_{h} \cdot v * \mathbf{e}_{\partial \Omega} \cdot\left(\nabla_{h} p\right)_{\partial \Omega}=0
$$

A TVD Runge-Kutta method [22] is used for time integration of both advections eqn. (4) and re-initialization eqn. (5). Solving re-initialization eqn. (5) to steady-state leads to a smooth transition of $\phi_{i}$ at the interface, proportional to the diffusion coefficient, $\varepsilon=0.5 h^{0.9}$, with $\mathrm{h}$ defined as the grid size [5]. From the numerical experiments, it is concluded that one iteration per physical time step is enough to solve the re-initialization eqn. (5) for steady state, and to maintain a constant thickness of the CLS functions [4, 5].

The aforementioned numerical algorithms are implemented in the framework of an in-house parallel $\mathrm{C}++$ /MPI code called TermoFluids. The code is run on the supercomputer MareNostrum III using a range of 128-256 CPU-cores for 3D simulations of both single and 
two droplets, up to 512 CPU-cores for 3D simulations of multiple falling droplets. The reader is referred to Refs $[4-7,11]$ for validations, verifications and further technical details on the finite-volume discretization of the Navier-Stokes equations, energy equation and CLS equations on 3D unstructured grids.

\section{NUMERICAL EXPERIMENTS AND DISCUSSION}

Nondimensionalization of the relevant physical quantities in the gravity-driven motion of bubbles and droplets can be summarized as $[5,7,14]$ :

$$
\mathrm{Mo}=\frac{g \mu_{c}{ }^{4} \Delta \rho}{\rho_{c} \sigma^{3}}, \mathrm{Eo}=\frac{g d^{2} \Delta \rho}{\sigma}, \eta_{\rho}=\frac{\rho_{d}}{\rho_{c}}, \eta_{\mu}=\frac{\mu_{d}}{\mu_{c}} .
$$

where $M o$ is the Morton number, Eo is the Eötvös number, $R e$ is the Reynolds number, $\eta_{\rho}$ is the density ratio, $\eta_{\mu}$ is the viscosity ratio, sub index $c$ is used to denote the continuous fluid and $d$ the droplet fluid, $\Delta \rho=\left|\rho_{d}-\rho_{c}\right|$ and $U_{T}$ is the terminal velocity of the droplet. The confinement ratio is defined as $C R=L / d$, where $d$ is the initial droplet diameter and $L$ is the characteristic length of the domain, which in square channels corresponds to the square side. The results are presented in terms of the dimensionless time $t^{*}=t g^{1 / 2} d^{-1 / 2}$ and Reynolds number $\operatorname{Re}(t)=\rho_{c} \boldsymbol{e}_{x} \cdot \boldsymbol{v}_{c}(t) d / \mu_{c}$ where $\boldsymbol{v}_{c}(t)$ is the droplet velocity.

\subsection{Verification and validation}

The computational methods used in this work have been extensively validated against experiments and numerical results from the literature [4-7, 11], for instance: 2D dam-break [5], 2D and $3 \mathrm{D}$ rising bubbles $[5,7,11]$, droplet deformation in a shear flow [11], and static bubble [11]. Particularly, the ability of the multiple marker CLS method to capture the hydrodynamics in the thin film formed during the collision of fluid particles without coalescence has been proven to be accurate for the droplet collision against a fluid-fluid interface [4], binary droplet collision with bouncing outcome [4], and thermocapillary-driven motion of fluid particles [6]. Therefore, this research can be considered as a further step in the understanding of the physics of falling droplets in a vertical channel, with the aid of the multiple marker CLS method introduced by [4].

The falling motion of a droplet is simulated in a circular channel with $C R=8$, height $H=12 d$ (y-axis) and diameter $D=8 d(x-z$ plane $)$. The droplet initially located at $(x, y, z)=(0,10 d, 0)$. A non-uniform hexahedral mesh of $4.4 \mathrm{M}$ control volumes is used, with uniform grid size $h=d / 30$ concentrated around the $y$-axis, which is aligned with the gravity g. The dimensionless parameters are $E o=6.4, M o=1.03 \times 10^{-5}, \eta_{\rho}=1.19$ and $\eta_{\mu}=0.33$ which correspond to those used in the experiment of Ref. [24]. Figure 1 shows a comparison of $R e$ calculated by the CLS method $[4,5]$ employed in this research, against the results obtained using a coupled volume-of-fluid/level-set method (VOFLS) introduced in our previous work [11], and experimental Reynolds number reported by [24]. These results confirm the accuracy of the approach used in this research.

\subsection{Single droplets falling in a vertical channel.}

Now, the effect of the wall on the falling motion of the droplet is researched. The computational domain is a square channel with height $H=12 d$ (y-axis) and square side $D=2 d(x-z$ 

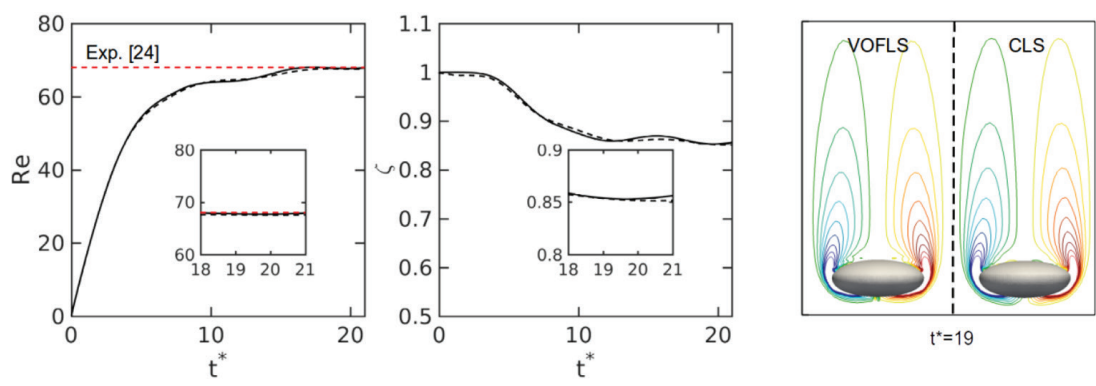

Figure 1: $E o=6.4, M o=1.03 \times 10^{-5}, \eta_{\rho}=1.19, \eta_{\mu}=0.33$. Re computed by CLS method [5] used in this work (continuous black line), VOFLS [11] (- - ), experiment of [24] (red line). Sphericity $\left(\zeta=\pi d^{2} / \iiint\|\nabla \phi\| d V\right)$. Vorticity $\boldsymbol{e}_{x} \cdot(\nabla \times \boldsymbol{v})$ on the plane $y$-z.

plane), thus the confinement ratio is $C R=2$. A cartesian mesh of $80 \times 80 \times 480$ grid elements is used in present simulations, which corresponds to the grid size $h=d / 40$. The selected dimensionless parameters are $E o=\{0.5,2.5,5.0\}, M o=\left\{10^{-5}, 10^{-6}, 10^{-7}, 10^{-8}\right\}, \eta_{\rho}=1.2$, $\eta_{\mu}=1.0$. At the beginning, both drop and continuous fluids are quiescent, whereas the droplet centroid is located slightly deviated from the symmetry $y$-axis of the channel, in the position $(x, y, z)=(-0.125 d, 11 d, 0)$. The imposed boundary conditions are no-slip on the lateral walls and periodic along the vertical direction aligned with the gravity (y-axis). According to previous mesh refinement studies reported in Refs [5, 7], a grid resolution $\mathrm{h}=\mathrm{d} / 30$ is enough for the accurate simulation of buoyancy-driven rising bubbles at low and intermediate Reynolds numbers, $\mathrm{O}(100)$, whereas the grid size $\mathrm{h}=\mathrm{d} / 35$ was used in Ref [4] to capture the thin layer formed during the collision of a droplet with a fluid interface in the context of the multiple marker CLS method used in this work. Thus, the grid resolution $\mathrm{h}=\mathrm{d} / 40$ is used unless otherwise stated.

Figure 2 shows that the droplet migrates to the symmetry axis of the channel for $E o=0.5$ and $10^{-7} \leq M o \leq 10^{-5}$; furthermore, when the droplet achieves the symmetry axis, the lateral velocity $U^{*}$ vanished, whereas for $M o \leq 10^{-8}$ the droplet centroid oscillates around the $y$-axis. For $E o=2.5$ and $10^{-5} \leq M o \leq 10^{-6}$, the droplet migrates to the symmetry axis of the channel. However, damped oscillations arise before the lateral velocity $U^{*}$ disappears. These oscillations are more evident as $M o$ increases for $10^{-8} \leq M o \leq 10^{-7}$. Finally for $E o=5$ droplet deformation effects appear, and the trajectories in Fig. 1 shows that the droplets achieve an equilibrium position at the symmetry axis of the channel after few oscillations, $10^{-5} \leq M o \leq 10^{-6}$, whereas the droplet never reaches an equilibrium position for $M o \geq 10^{-7}$. A chaotic behaviour is observed for $E o=0.5$ and $M o=10^{-8}$. Figure 3 shows the time evolution of $R e$, which demonstrates that droplets achieve a non-oscillatory terminal velocity, except for $(E o, M)=\left\{\left(2.5,10^{-8}\right),\left(5,10^{-7}\right),\left(5,10^{-8}\right)\right\}$ which present oscillatory terminal velocities. Figure 4 depicts the vorticity $\boldsymbol{e}_{x} \cdot(\nabla \times v)$, on the plane $y$ - $z$ produced by the interaction of the droplets with the wall, where a turbulent flow can be distinguished for $(E o, M)=\left(5,10^{-8}\right)$.

\subsection{Two droplets falling in a vertical channel}

The interaction of two droplets is investigated in this section. The spatial domain is a square channel with height $H=12 d$ (y-axis) and square side $H=4 d$ ( $x$-z plane). Thus, the 

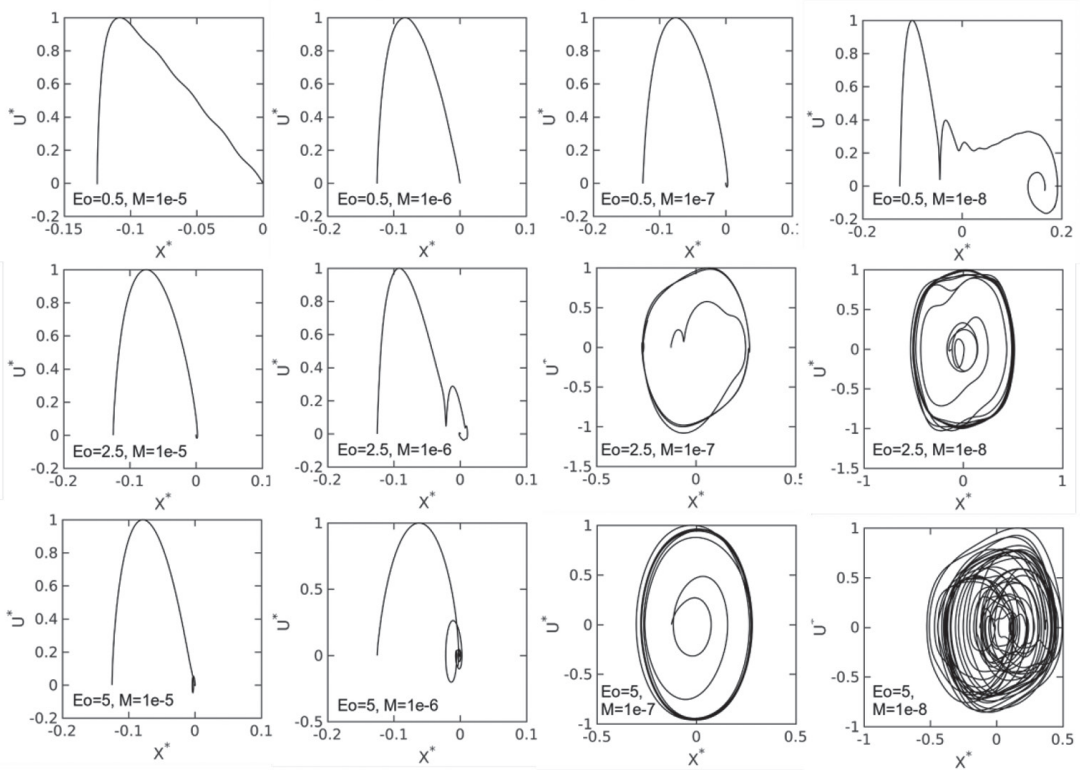

Figure 2: The lateral migration velocity $U^{*}=\boldsymbol{e}_{x} \cdot \boldsymbol{v}_{\boldsymbol{c}}(t) / \max \left\{\boldsymbol{e}_{x} \cdot \boldsymbol{v}_{\boldsymbol{c}}(t)\right\}$ of the droplet versus the lateral position $X^{*}=x / d$.
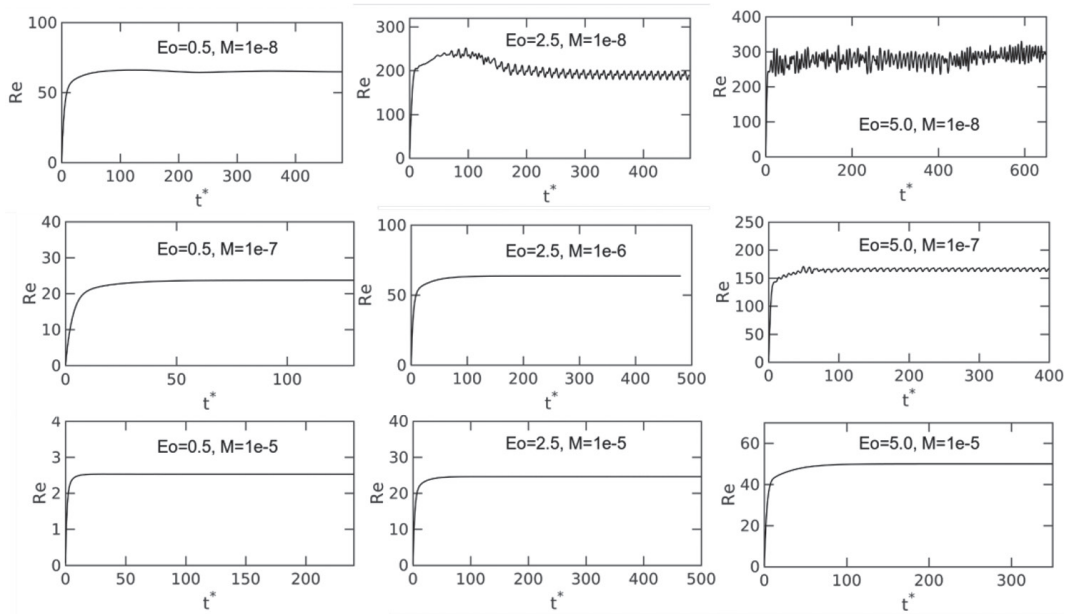

Figure 3: $R e(t)$ for a single droplet at $0.5 \leq E o \leq 5,10^{-8} \leq M o \leq 10^{-5}, \mathrm{CR}=2$.

confinement ratio is $C R=4$. A cartesian mesh of $160 \times 160 \times 480$ grid elements is used in the present research, which leads to the grid size $h=d / 40$. The selected dimensionless parameters are $(E o, M o)=\left\{\left(0.5,10^{-6}\right),\left(0.5,10^{-7}\right),\left(2.5,10^{-7}\right)\right\}, \quad \eta_{\rho}=1.2, \quad \eta_{\mu}=1.0$. At the beginning, both droplets are aligned along the $y$-axis, whereas the droplet centroids are located in the positions $\boldsymbol{x}_{1}=(0,11 d, 0)$ and $\boldsymbol{x}_{2}=(0,9.5 d, 0)$. The Reynolds number, centroid-centroid distance $\left(s=\left\|\boldsymbol{x}_{2}-\boldsymbol{x}_{1}\right\| / d\right)$, and configuration angle $(\theta)$ between the plane orthogonal to the $y$-axis and the vector $\Delta \boldsymbol{x}=\boldsymbol{x}_{2}-\boldsymbol{x}_{1}$ are used to follow the motion of the 

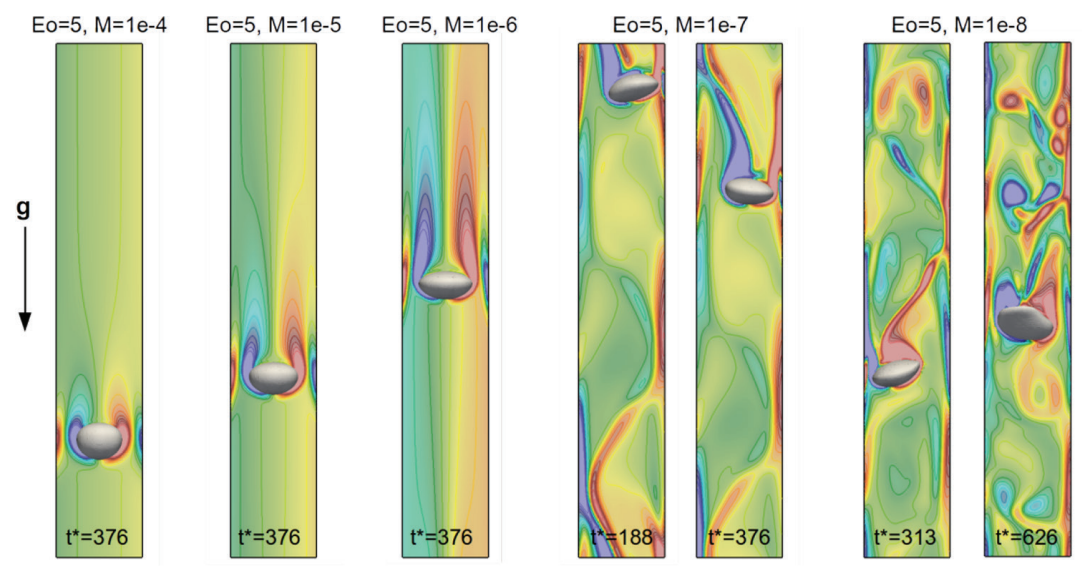

Figure 4: Vorticity $\boldsymbol{e}_{x} \cdot(\nabla \times \boldsymbol{v})$ on the plane $y-z$ produced by the wall-droplet interaction.

droplets. Figure 5 illustrates the different stages of the interaction of two droplets, which is similar to the so-called drafting-kissing-tumbling (DKT) mechanism reported by [10, 24]. In the drafting stage $\left(t^{*}=6.3\right)$, the wake generated by the leading droplet accelerates the falling motion of the trailing droplet, until the trailing droplet touch the leading droplet $\left(t^{*}=\{12.5,18,8\}\right)$ during the kissing stage. Then, the droplets rotate and separate each other $\left(t^{*}=\{25.1,31.3,37.6\}\right)$ in the tumbling stage. Finally, the droplets interact with the wall, but they do not collide with it, where a bouncing effect is observed $\left(t^{*}=\{43.9,50.1\}\right)$. Figure 6 depicts the time evolution of Re, centroid-centroid distance (s) and configuration angle $(\theta)$. After the DKT interaction, a stable motion is achieved by the two droplets for $(E o, M o)=\left(0.5,10^{-6}\right)$. Moreover, the falling motion of the droplets continues with a sideby-side configuration. As the $M o$ increases, for $(E o, M o)=\left(0.5,10^{-7}\right)$, the Reynolds number increase and an oscillatory behaviour is observed previous the droplets achieves a steady state similar to the previous case. For $(E o, M o)=\left(0.5,10^{-7}\right)$, surface tension decreases and the deformation of the droplets introduce more complexity to the interaction, and eventually, an oscillatory motion is observed after the DKT stage. These results are consistent with front-tracking simulations reported in Ref [20].
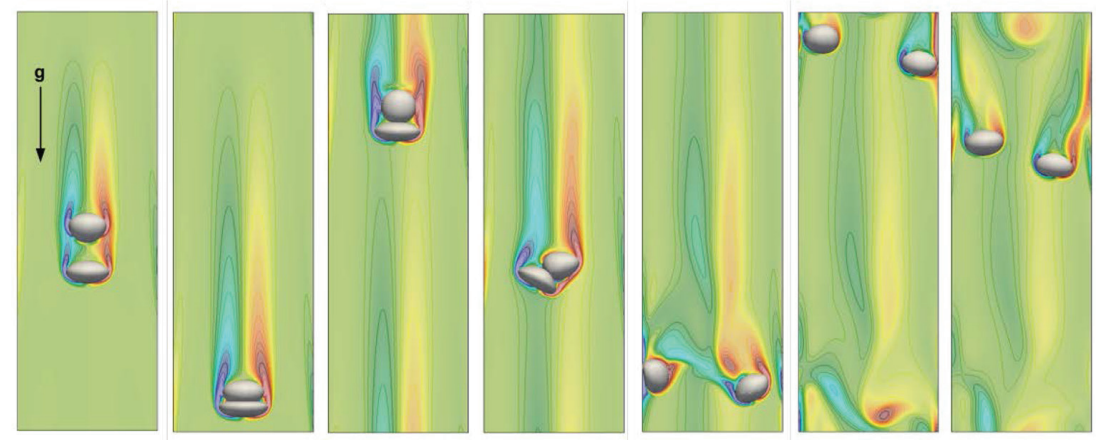

Figure 5: Vorticity $\boldsymbol{e}_{x} \cdot(\nabla \times \boldsymbol{v})$ on the plane $y-z$ and the interaction of two droplets for $E o=2.5, M o=10^{-7}$, and $t^{*}=\{6.3,12.5,18.8,25.1,31.3,37.6,43.9,50.1\}$. 

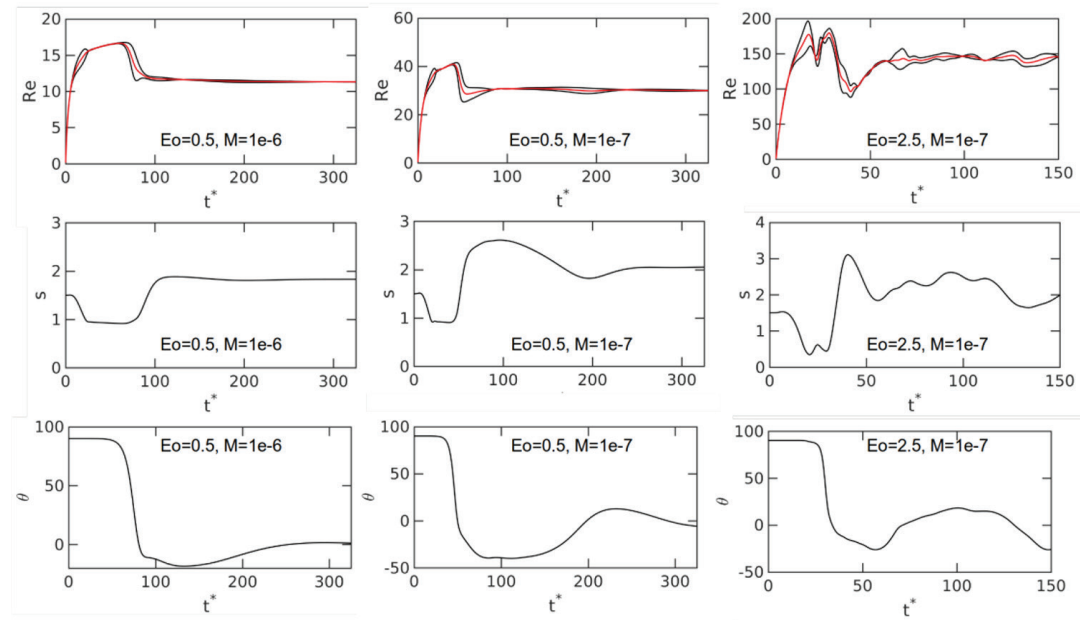

Figure 6: Interaction of two droplets. Time evolution of Re, centroid-centroid distance ( $\left.s=\left\|\boldsymbol{x}_{2}-\boldsymbol{x}_{1}\right\| / d\right)$, and configuration angle $(\theta)$.

\subsection{Multiple droplets falling in a vertical channel}

The interaction of multiple droplets is investigated in this section. The computational domain and the grid configuration are same as in the previous section, with grid size $h=d / 40$. Figure 7 depicts the interaction of four droplets initially aligned on the $y$-axis, with centroid-centroid separation $1.5 \mathrm{~d}$. The dimensionless parameters are $(E o, M o)=\left(2.5,10^{-5}\right)$, $\eta_{\rho}=1.2, \eta_{\mu}=1.0$. Complex interactions are observed for this case, but basically, these are also based on the DKT mechanism, in which the wake of the leading droplets attracts the second droplet $\left(t^{*}=31.3\right)$. Then, the combined wake attracts the third droplet until the three droplets are in contact $\left(t^{*}=31.3\right)$. However, the wake of these three droplets does not attract the fourth droplet, which moves slower that the set of droplets. The configuration of three droplets in contact and vertical alignment is unstable, and this arrangement breaks $\left(t^{*}=37.6\right.$ ). Finally, the four droplets separate each other $\left(t^{*}=62.6\right)$ and continue their motion without colliding with the wall. Figure 8 shows that the four droplets achieve a steady-state vertical velocity (see $R e$ ), whereas the distance between the wall and the droplets (see $X^{*}$ and $Y^{*}$ )

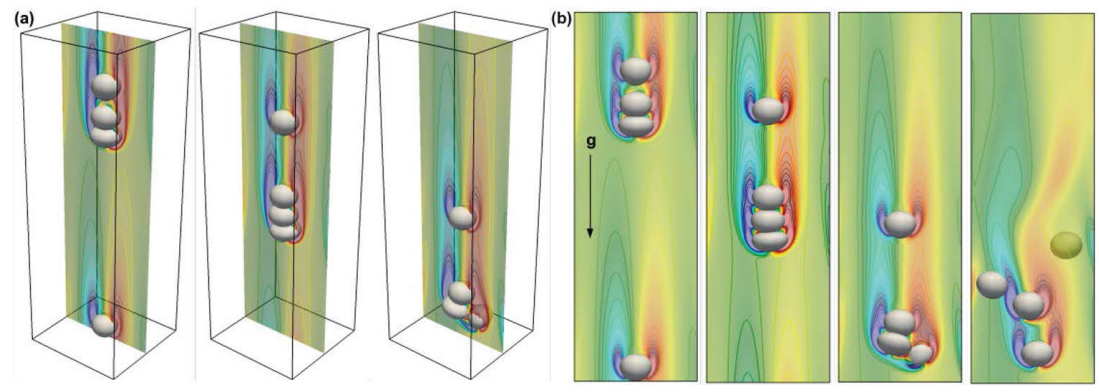

Figure 7: Vorticity $\boldsymbol{e}_{x} \cdot(\nabla \times \boldsymbol{v})$ on the plane $y-z$. Interaction of four droplets for $E o=2.5, M o=10^{-5}$. (a) $t^{*}=\{25.1,31.3,37.6\}$. (b) $t^{*}=\{25.1,31.3,37.6,62.6\}$. 

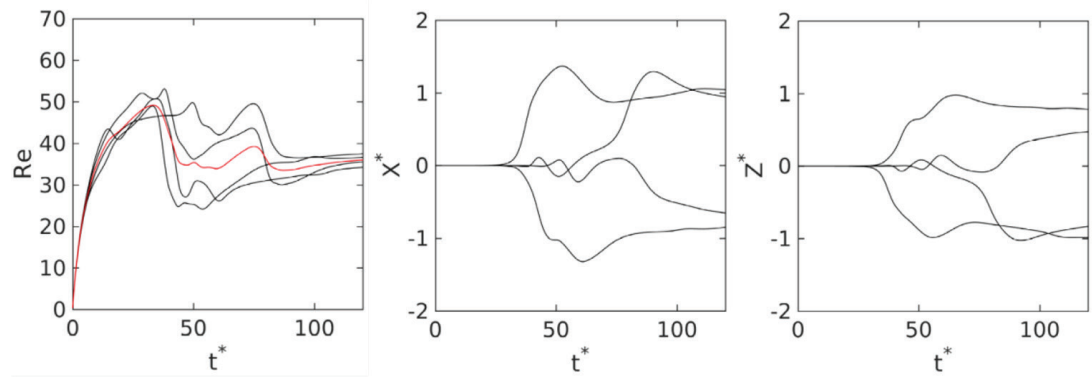

Figure 8: Interaction of four droplets, $E o=2.5, M o=10^{-5}$. Time evolution of $R e, X^{*}=x / d$ and $Z^{*}=z / d$. Red line is used for the average $R e$.

tend to be constant as the time advances. Finally, Figs. 9 and 10 depict the interaction of 18 droplets initially placed in two horizontal layers of nine droplets following a random pattern, for $(E o, M o)=\left(2.5,10^{-5}\right)$.

In the beginning, the two droplet layers separate each other; however, one of the droplets separates from the group. Then, the wake interactions break the configuration of two layers, and the droplets begin to interact with each other following the DKT mechanism when the
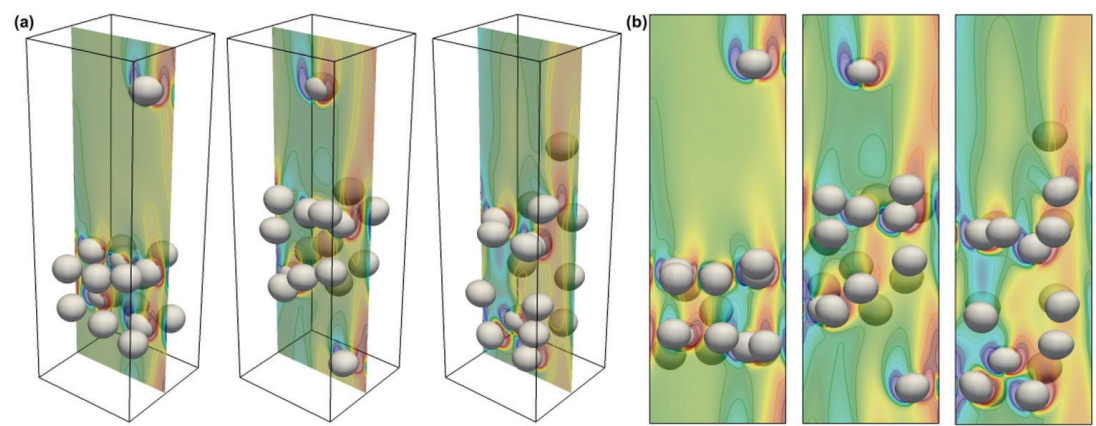

Figure 9: Vorticity $\boldsymbol{e}_{x} \cdot(\nabla \times \boldsymbol{v})$ on the plane $y$-z. Interaction of four droplets for $E o=2.5, M o=10^{-5}$. (a) $t^{*}=\{37.6,75.2,112.8\}$. (b) $t^{*}=\{37.6,75.2,112.8\}$.
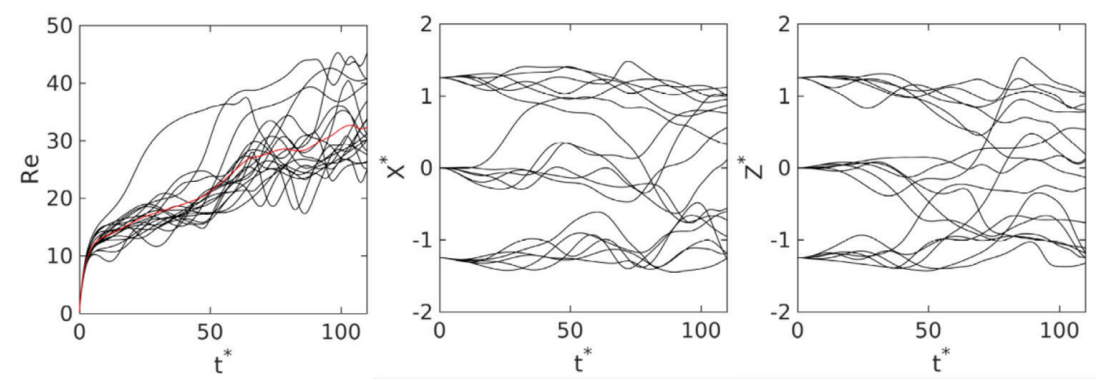

Figure 10: Interaction of four droplets, $E o=2.5, M o=10^{-5}$. Time evolution of $\operatorname{Re}, X^{*}=x / d$ and $Z^{*}=z / d$. Red line is used for the average $R e$. 
droplets approach in the vertical direction, whereas a bouncing effect is observed between the wall and the droplets aligned horizontally. For this flow condition, the droplets do not touch the wall.

\section{CONCLUSIONS}

In this work, Direct Numerical Simulations of falling droplets in a vertical channel have been performed, using the multiple marker CLS method introduced in Refs $[4,6]$. These numerical experiments show the capacity of the method for the simulation of single and multiple droplets, including their interactions and collisions, avoiding the numerical coalescence of the droplets. Some physical insights can be obtained from these simulations for the flow conditions analysed in this work. The interaction of a single drop with the wall leads to a stable motion for low Eo and high Mo. As the Eo increases or Mo decreases, the interaction of the droplet with the wall lead to an unstable motion, due to the effects of deformability and high Reynolds numbers. The interaction of two droplets initially aligned in vertical positions follows the DKT mechanism. Furthermore, the same mechanism was observed in the case of four droplets vertically aligned. For these flow regimes, the droplets do not touch the wall, but a bouncing interaction was observed. These interactions are also observed in the falling motion of multiple droplets, in which the DKT mechanism is followed for droplets in vertical alignment, and bouncing effect arises for droplets in horizontal alignment. Future work includes the incorporation of complex transport phenomena, such as the effect of surfactants, gas-liquid phase change, heat and mass transfer.

\section{ACKNOWLEDGEMENTS}

This work has been financially supported by the Ministerio de Economía y Competi- tividad, Secretaría de Estado de Investigación, Desarrollo e Innovación (MINECO), Spain (ENE2015-70672-P), and by Termo Fluids S.L. Néstor Balcázar acknowledges financial support of the Programa Torres Quevedo, MINECO (PTQ-14-07186), Spain. Three dimensional simulations were carried out using computer time awarded by PRACE $10^{\text {th }}$ Call (Project 2014112666) on the supercomputer MareNostrum III based in Barcelona, Spain.

\section{REFERENCES}

[1] Tryggvason, G., Dabiri, S., Abouhasanzadeh, B. \& Lu, J., Multiscale considerations in direct numerical simulations of multiphase flows. Physics of Fluids, 25, 031302, 2013. https://doi.org/10.1063/1.4793543

[2] Tryggvason, G., Bunner, B., Esmaeeli, A., Juric, D., Al-Rawahi, N., Tauber, W., Han, J., Nas, S. \& Jan, Y-J., A front-tracking method for the computations of multiphase flow. Journal of Computational Physics, 169, pp. 708-759, 2001.

https://doi.org/10.1006/jcph.2001.6726

[3] Fakhari, A. \& Rahimian, M.H., Simulation of falling droplet by the lattice Botzmann method. Communications in Nonlinear Science and Numerical Simulation, 14, pp. 3046-3055, 2009. https://doi.org/10.1016/j.cnsns.2008.10.017

[4] Balcázar, N., Lehmkhul, O., Rigola, J. \& Oliva, A., A multiple marker level-set method for simulation of deformable fluid particles. International Journal of Multiphase Flow, 74, pp. 125-142, 2015. https://doi.org/10.1016/j.ijmultiphaseflow.2015.04.009 
[5] Balcázar, N., Jofre, L., Lehmkhul, O., Castro, J. \& Rigola, J., A finite-volume/levelset method for simulating two-phase flows on unstructured grids. International Journal of Multiphase Flow, 64, pp. 55-72, 2014.

https://doi.org/10.1016/j.ijmultiphaseflow.2014.04.008

[6] Balcázar, N., Rigola, J., Castro, J. \& Oliva, A., A level-set model for thermocapillary motion of deformable fluid particles. International Journal of Heat and Fluid Flow, 62 , pp. 324-343, 2016.

https://doi.org/10.1016/j.ijheatfluidflow.2016.09.015

[7] Balcázar, N., Lemhkuhl, O., Jofre, L. \& Oliva, A., Level-set simulations of buoyancydriven motion of single and multiple bubbles. International Journal of Heat and Fluid Flow, 56, pp. 91-107, 2015.

https://doi.org/10.1016/j.ijheatfluidflow.2015.07.004

[8] Olsson, E. \& Kreiss, G., A conservative level set method for two phase flow. Journal of Computational Physics, 210, pp. 225-246, 2005.

https://doi.org/10.1016/j.jcp.2005.04.007

[9] Sussman, M., Smereka, P. \& Osher, S. A level set approach for computing solutions to incompressible two-phase flow. Journal of Computational Physics, 144, pp. 146-159, 1994.

https://doi.org/10.1006/jcph.1994.1155

[10] Hirt, C. \& Nichols, B., Volume of fluid (VOF) method for the dynamics of free boundary. Journal of Computational Physics, 39, pp. 201-225, 1981.

https://doi.org/10.1016/0021-9991(81)90145-5

[11] Balcázar, N., Lehmkhul, O., Jofre, L., Rigola, J. \& Oliva, A., A coupled volume-offluid/levelset method for simulation of two-phase flows on unstructured meshes. Computers and Fluids, 124, pp. 12-29, 2016.

https://doi.org/10.1016/j.compfluid.2015.10.005

[12] Coyajee, E. \& Boersma, J.B, Numerical simulation of drop impact on a liquid-liquid interface with a multiple marker front-capturing method. Journal of Computational Physics, 228, pp. 4444-4467, 2009.

https://doi.org/10.1016/j.jcp.2009.03.014

[13] Sussman, M. \& Puckett, E.G., A coupled level set and volume-of-fluid method for computing 3D and axisymmetric incompressible two-phase flows. Journal of Computational Physics, 162, pp. 301-337, 2000.

https://doi.org/10.1006/jcph.2000.6537

[14] Clift, R., Grace, J.R. \& Weber, M.E., Bubbles, drops and particles. Academic Press, New York, 1978.

[15] Moore, D.W., The rise of a gas bubble in a viscous fluid. Journal of Fluid Mechanics, 6, pp. 113-130, 1959. https://doi.org/10.1017/s0022112059000520

[16] Taylor, T.D. \& Acrivos, A., On the deformation and drag of falling viscous drop at low Reynolds number. Journal of Fluid Mechanics, 18, pp. 466-476, 1964. https://doi.org/10.1017/s0022112064000349

[17] Brignell, A.S., The deformation of a liquid drop at small Reynolds number. Quarterly Journal of Mechanics and Applied Mathematics, 26, pp. 99-107, 1973. https://doi.org/10.1093/qjmam/26.1.99 
[18] Han, J. \& Tryggvason, G., Secondary breakup of axisymmetric liquid drops I. Acceleration by a constant body force. Physics of Fluids, 11(12), pp. 3650-3667, 1999. https://doi.org/10.1063/1.870229

[19] Mortazavi, S. \& Tryggvason, G., A numerical study of the motion of drops in Poiseuille flow. Part 1. Lateral migration of one drop. Journal of Fluid Mechanics, 41, pp. 325-350, 2000.

https://doi.org/10.1017/s0022112099008204

[20] Amiri, M. \& Mortazavi, S., Three-dimensional numerical simulation of sedimenting drops inside a vertical channel. International Journal of Multiphase Flow, 56, pp. 40-53, 2013.

https://doi.org/10.1016/j.ijmultiphaseflow.2013.05.007

[21] Brackbill, J.U., Kothe, D.B. \& Zemach, C., A continuum method for modeling surface tension. Journal of Computational Physics, 100, pp. 335-354, 1992.

https://doi.org/10.1016/0021-9991(92)90240-y

[22] Gottlieb, S. \& Shu, C.W., Total variation dimishing Runge-Kutta schemes. Mathematics of Computations, 67, pp. 73-85, 1998.

https://doi.org/10.1090/s0025-5718-98-00913-2

[23] Chorin, A.J., Numerical solution of the Navier-Stokes equations. Mathematics of Computation, 22, pp. 745-762, 1968.

https://doi.org/10.1090/s0025-5718-1968-0242392-2

[24] Mohamed-Kassim, Z. \& Longmire, E.K., Drop impact on a liquid-liquid interface. Physics of Fluids, 15, pp. 3263-3273, 2003.

https://doi.org/10.1063/1.1609993

[25] Feng, J., Hu, H.H. \& Joseph, D.D., Direct simulation of initial value problems for the motion of solid bodies in a Newtonian fluid Part 1. Sedimentation. Journal of Fluid Mechanics, 261, pp. 95-134, 1994.

https://doi.org/10.1017/s0022112094000285 